Адрес статьи / To link this article: $\underline{\mathrm{http}: / / \mathrm{cat} .1 \mathrm{fmo.ru} / \mathrm{ru} / 2019 / \mathrm{v} 4-\mathrm{i} 1 / 170}$

\title{
Погружение как один из ключевых факторов успешных проектов в игровой индустрии
}

\author{
Н.М. Бортейн ${ }^{1}$, А.А. Смолин ${ }^{1,2}$ \\ ${ }^{1}$ Университет ИТМО, Россия \\ ${ }^{2}$ Санкт-Петербургский государственный университет, Россия \\ naranbortein@gmail.com, smolin@itmo.ru
}

\begin{abstract}
Аннотация. В статье исследуется феномен погружения в контексте видеоигр, представляющий собой особый вид пользовательского опыта. Авторами рассмотрен вопрос определения термина «погружение», описаны факторы погружения.
\end{abstract}

Ключевые слова: погружение, видеоигры, компьютерные игры, гейм-дизайн

Погружение - сложное явление, которым обычно описывают опыт пользователя в контексте исследования развлечений, в том числе видеоигр. Данная концепция широко используется игроками, разработчиками видеоигр, журналистами и исследователями, но единого понимания того, к каким переживаниям или психологическим явлениям это на самом деле относится, нет. Схожим с погружением понятием можно считать феномен «присутствия» (англ. presence), который используется с целью оценки так называемой иммерсивности (англ. immersion - погружение) системы. Понятие относится к психологическому опыту, заключающемуся в ощущении присутствия в мире, порожденном компьютером. [1]. Подобным образом о погружении говорит Джанет Мюррей, которая определила погружение как «ощущение окружения совершенно другой реальностью, которая захватывает все наше внимание, все наше аппарат восприятия» [2]. Соотнеся данные определения, можно сделать предположение, что погружение и присутствие являются достаточно близкими понятиями, чтобы использовать их как синонимы, однако это не так. Причиной этому является тот факт, что термин «присутствие» первоначально был разработан в контексте телеопераций и он, в значительной степени, зависит от метафоры транспорта, а в контексте видеоигр всё же предпочтительнее использовать термин «погружение».

За всё время изучения исследователями было дано несколько определений данного термина [3]. Менетта и Блейд описывают погружение как эмоциональный отклик, представленный в виртуальной среде. Куманс и Тиммерманс определяли погружение как «чувство глубокой вовлеченности, состояние, когда люди входят в воображаемый мир, как будто он реальный». В свою очередь Макмахан определил погружение как степень погружения со степенью взаимодействия между игроком и игровым миром, особо ответив реализм как один из определяющих составляющих структуры погружения [4].

У разработчиков видеоигр имеется множество инструментов для создания благоприятных условий для игрока. Стоит отметить, что опыт погружения может создать исключительно личные 
и эмоциональные моменты. Проводились исследования, которые установили его терапевтический характер [5].

Погружение создается из взаимодействия между игроком и игровой системой. Игроки активно участвуют в построении игрового опыта, принося их личный опыт, ожидания и желания вместе с ними [6]. Существует множество факторов, которые могут повлиять на погружение игрока, и которые не зависят от разработчиков, например, обстоятельства места и времени игровой сессии и состояние психического здоровья человека, но в любом случае ключевым компонентом в содействии погружению в видеоиграх является их интерактивность. Погружение может происходить в книгах или фильмах без какого-либо взаимодействия между объектом и потребителем. Интерактивность же дает видеоиграм уникальную возможность привлечь игрока к формированию и разрешению различных игровых ситуаций. Похожим образом погружение описывал Христу, называя его эмерджентным атрибутом, который генерируется из взаимодействия между пользователем и видеоигрой [7]. Интерактивность может относиться к вовлечению игрока во многих отношениях.

Относительно мало известно о том, как пользовательский опыт изменяется в зависимости от жанра игры в связи с особенностями различных категорий пользователей. Проводились исследования, изучающие мотивы игроков, в результате которых было выявлено, что пользователи онлайн-игр, в основном шутеров от первого лица, были более мотивированы достижениями, в то время как пользователи, отдающие предпочтение жанру ролевых игр, были более мотивированы погружением [8].

Так как погружение создается из взаимодействия между игроком и игровой системой, факторы, заложенные в формирование погружения, могут быть внешними и внутренними (пользовательскими).

Погружение может происходить на разных уровнях видеоигры как комплексного произведения: через нарратив, контролируемые и неконтролируемые игровые ситуации, психологические приемы, различные приемы геймдизайна и аудиовизуальные образы. Данные подходы не являются взаимоисключающими, а, наоборот, могут оказывать комплексное влияние на пользователя.

Нарратив (лат. narrare - рассказывать, повествовать; означает языковой акт, то есть вербальное изложение - в отличие от представления) - это повествование об определенной цепочке событий, вымышленных или реальных. Аспекты повествования включают в себя то, каким образом рассказывается история, какие события описывает, в каком контексте, и конструкцию всей истории.

Доступные играм способы для этой цели можно условно разделить на две основные категории: заложенное в игру повествование вкупе со сценарием и генерируемые в результате взаимодействия пользователя с игровым миром игровых ситуаций [9].

По словам Брайанта и Джильо, игровые сюжеты позволяют формировать у игроков глубокую эмоциональную привязанность к игровому миру и его персонажам, которая заставит их возвращаться к игре снова и снова [10]. Хорошее повествование может оправдать действия игрока, поставить для него цели. Существует множество различных повествовательных инструментов, которые можно использовать для создания погружения. Согласно Цинь игровые сюжеты обладают тремя характеристиками, которые отличают их от более традиционных медиапродуктов. Эти аспекты: интерактивность, нелинейная структура и взаимодействие между игровым процессом и историей [11].

Генерируемые в результате взаимодействия пользователя с игровым миром игровые ситуации также тесно связаны с интерактивностью игр. Повествование в данном случае основано на том, что разработчики оставляют множество взаимосвязанных друг с другом деталей общего сюжета, однако не рассказывают историю напрямую, оставляя пользователю возможность узнать все самим путем исследования игрового мира и сбором информации небольшими фрагментами с последующим осмыслением их взаимосвязи.

International Culture \& Technology Studies, Vol. 4, No. 1 
Игровые персонажи также могут оказать существенное влияние на игровой сюжет. В игре с контролируемым игроком персонажем, роль персонажа в привлечении игрока может быть чрезвычайно ценной. Чем более близок игроку его герой, тем сильнее эмоции, которые может вызвать история [9].

Настройка персонажа может предложить игроку сильные чувства идентификации. Во многих современных ролевых видеоиграх игрок может настроить своего персонажа так, как ему нравится, изменяя внешний вид, характер, биографию. Эти виды персонажей часто называют аватарами.

Кастомизация позволяет игроку больше идентифицировать себя с персонажем, усиливая мотивацию к игре [12]. Интересно то, что в противовес этому заявлению, Брайант и Джильо утверждают, что игрок становится более эмоционально вовлеченным в игру, когда он играет предварительно созданным персонажем, а не аватаром. Эти различия можно объяснить разной мотивацией игроков. Игроку, предпочитающему ролевые игры, больше нравится играть аватаром, в то время как игрок, который любит повествовательные элементы игры, будет более эмоционально вовлечен, играя за предварительно созданного персонажа.

Так или иначе, персонажи могут оказывать удивительно сильное влияние на погружение игрока - противоречие между мотивами игрока и игрового персонажа может создавать проблемы при формировании погружения. Ибистер в своей работе обсуждает важность познавательного погружения в построении сильных персонажей. Игрок должен быть в состоянии синхронизировать возможности решения проблем с возможностями игрового персонажа, иначе он испытает диссонанс, который сведет все погружение на нет [13]. Людонарративный диссонанс это противоречие между игроком, игровым дизайном и/или повествовательными элементами. Когда игровой процесс и мотивации игрока и персонажа синхронизированы, игрок может испытать то, что называется людонарративной гармонией.

Геймдизайн может обеспечивать погружение только в связи с нарративом. Без нарратива остается только поток, который в данном случае возникает без погружения. Наглядным примером могут послужить классические шахматы. В шахматах нет игрового мира, внутриигровой истории, нет персонажей, есть только цель, набор фигур, правил и игровой цикл.

Не у каждой игры есть четкая цель. Эти игры часто творческие, без условий победы, однако игроки часто устанавливают свои личные цели, наличие которых помогает им ощущать свободу собственного опыта. Так как нет цели и, соответственно, ценной награды, встроенной в игру, следовательно, наградой является личное удовлетворение. Даже если награды или цели не связаны строго с погружением игрока, они все еще могут играть важную роль в формировании погружения. Наличие привлекательных цели и награды мотивируют пользователя на постоянное повторение игрового цикла, что положительно сказывается как на потоке, так и на погружении [14].

Восприятие аудиовизуальных образов - один из самых важных и сильных внешних факторов. Визуальные эффекты, звуки и музыка оказывают большое влияние на погружение игрока в видеоигры. Они поддерживают рассказываемую историю, управляют эмоциями зрителя и создают впечатляющие кинематографические моменты. Они также могут иметь более практическое назначение, особенно в проектировании пользовательского интерфейса - играть важную роль в информировании игрока о различных игровых событиях. Визуальная иерархия в таком случае, например, используется для обеспечения того, чтобы важные части информации в игре становились более заметными для игрока [15]. Эта практика важна, так как игрок может сосредоточиться на более важных визуальных подсказках и не отвлекаться на поиск недостающей информации.

Звуки и музыка, помимо эстетического и эмоционального вклада, тоже являются частью интерфейса и эффективным способом информирования игрока об игровом мире. Определенные звуки могут указывать игроку о состоянии его персонажа и окружающего пространства, сопровождают взаимодействие с графическим интерфейсом. Наличие звука является неотъемлемой частью погружения игрока [16].

Мировой нарратив состоит из визуальной и слуховой нарративной информации виртуальной игровой среды. Повествование о мире можно найти в архитектуре, письмах, 
записках, одежде и мебели, а также во многих других местах. Почти любой объект или его отсутствие в виртуальной среде может рассказать историю, что очень близко к классическому нарративу, однако больше касается построению правильного с точки зрения игрового дизайна виртуального пространства. Таким образом, повествование в игровом мире может создать погружение через пространственное и повествовательное участие.

Сильвестр предполагает, что лучший метод построения повествования в мире - через наличие или отсутствие деталей в игровой среде [29]. К примеру, умирающие комнатные растения или неоткрытое письмо могут указывать на длительное отсутствие жителей в доме. В кинематографе и театре расположение элементов сцены для формирования визуальной темы называется мизансценой. Ключевые аспекты мизансцены включают такие элементы, как: освещение, пространство и состав. Методы, используемые при построении мизансцены, также могут содержать применимые знания к проектированию игровых миров.

Согласованность в мире и выражение внутреннего связи внутри, усиливает эффект повествования мира. Непоследовательность в мире может помешать игроку и привести к отсутствию погружения.

\section{Подытожив вышесказанное можно сделать следующие выводы:}

- Погружение - понятие, часто связанное с крайне положительным пользовательским опытом. Такой опыт отмечается исследователями игр, как важный аспект взаимодействия человека $\mathrm{c}$ интерактивной человеко-компьютерной системой. Погружение концептуализируется как психологическое чувство причастности или ощущение, что пользователь на самом деле в процессе игре или игровом мире.

- Погружение может происходить на разных уровнях видеоигры как комплексного произведения: через нарратив, контролируемые и неконтролируемые игровые ситуации, психологические приемы, различные приемы игрового дизайна, аудиовизуальные образы. Данные подходы не являются взаимоисключающими, а наоборот, могут оказывать комплексное влияние на пользователя.

\section{Литература}

[1] Lombard, Matthew/Ditton, Theresa (1997): “At the Heart of It All: The Concept of Presence”, in: Journal of Computer Mediated Communication 3/2 [Электронный pecypc]: URL: http://jcmc.indiana.edu/vol3/issue2/lombard.html. (Дата обращения 08.06.2019);

[2] Murray, Janet (1997): Hamlet on the Holodeck. The Future of Narrative in Cyberspace, Cambridge/London: MIT. p 98;

[3] J. Huhtala, P. Isokoski, and S. Ovaska, "The usefulness of an immersion questionnaire in game development," in CHI'12 Extended Abstracts on Human Factors in Computing Systems, pp. 1859-1864, ACM, 2012.;

[4] P. Cairns, A. Cox, N. Berthouze, C. Jennett, and S. Dhoparee, "Quantifying the experience of immersion in games.," in CogSci 2006 Workshop: Cognitive Science of Games and Gameplay, 2006.;

[5] Baek, Y. 2013. Psychology of Gaming. Ebook. New York: Nova Publishers p. 8 [Электронный pecypc]: URL: https://ebookcentral.proquest.com (Дата обращения 08.06.2019);

[6] Ermi, L. \& Mäyrä, F. 2005. Changing Views: Worlds in Play. International Conference, p. 98 [Электронный pecypc]: URL: http://www.digra.org/digitallibrary/publications/fundamental-components-of-the-gameplayexperienceanalysing-immersion/ (Дата обращения 08.06.2019);

[7] Christou, G. 2013. The interplay between immersion and appeal in video games. Computers in Human Behavior 32, 92-100;

[8] Ghuman, D. and Griffiths, M. A Cross-Genre Study of Online Gaming. Int. Journal of CyberBehavior, Psychology and Learning 2, 1 (2012), 13-29;

[9] Skolnick, E. 2014. Video Game Storytelling: What Every Developer Needs to Know about Narrative Techniques. New York: Watson-Guptill Publications;

[10]Bryant, R. \& Giglio, K. 2015. Slay the dragon: writing great video games. California: Michael Wiese Productions p. 59;

[11] Qin, H. \& Rau, P. \& Salvendy, G. 2009. Measuring Player Immersion in the Computer Game Narrative. Journal of Human-Computer Interaction 25(2), 107-133;

[12] Teng, C. 2010. Customization, immersion satisfaction, and online gamer loyalty. Computers in Human Behavior 26, 1547-1554.;

International Culture \& Technology Studies, Vol. 4, No. 1 
[13] Ibister, K. 2006. Better Game Characters by Design : A Psychological Approach. Ebook. Florida: CRC Press. [Электронный ресурc]: URL: https://ebookcentral.proquest.com (Дата обращения: 08.06.2019);

[14]Calleja, G. 2014. In-Game : From Immersion to Incorporation. Ebook. London: The MIT Press. Available at: https://ebookcentral.proquest.com p.150;

[15] Sylvester, T. 2013. Designing Games A Guide to Engineering Experiences. California: O'Reilly p. 84;

[16] Collins, K. 2013. Playing with Sound: A Theory of Interacting with Sound and Music in Video Games. Ebook. London: The MIT Press., p. 58 [Электронный pecypc]: URL: https://ebookcentral.proquest.com (Дата обращения 08.06.2019)

\title{
Immersion as one of the key factors for successful projects in the gaming industry
}

\author{
N.M. Bortein ${ }^{1}$, A.A. Smolin ${ }^{1,2}$ \\ ${ }^{1}$ ITMO University, Russia \\ ${ }^{2}$ Saint-Petersburg State University, Russia
}

\begin{abstract}
The article explores the phenomenon of immersion in video games as a special kind of user experience. The authors considered the issue of defining the term "immersion" and described the immersion factors.
\end{abstract}

Keywords: immersion, videogame, computer game, game design

\section{References}

[1] Lombard, Matthew/Ditton, Theresa (1997): "At the Heart of It All: The Concept of Presence", in: Journal of Computer Mediated Communication 3/2, http://jcmc.indiana.edu/vol3/issue2/lombard.html.

[2] Murray, Janet (1997): Hamlet on the Holodeck. The Future of Narrative in Cyberspace, Cambridge/London: MIT. p 98

[3] J. Huhtala, P. Isokoski, and S. Ovaska, "The usefulness of an immersion questionnaire in game development," in CHI'12 Extended Abstracts on Human Factors in Computing Systems, pp. 1859-1864, ACM, 2012.

[4] P. Cairns, A. Cox, N. Berthouze, C. Jennett, and S. Dhoparee, "Quantifying the experience of immersion in games.," in CogSci 2006 Workshop: Cognitive Science of Games and Gameplay, 2006.

[5] Baek, Y. 2013. Psychology of Gaming. Ebook. New York: Nova Publishers. https://ebookcentral.proquest.com p. 8

[6] Ermi, L. \& Mäyrä, F. 2005. Changing Views: Worlds in Play. International Conference. Available at: http://www.digra.org/digitallibrary/publications/fundamental-components-of-the-gameplayexperienceanalysing-immersion/ $\mathrm{p} 98$.

[7] Christou, G. 2013. The interplay between immersion and appeal in video games. Computers in Human Behavior 32, 92-100.

[8] Ghuman, D. and Griffiths, M. A Cross-Genre Study of Online Gaming. Int. Journal of CyberBehavior, Psychology and Learning 2, 1 (2012), 13-29.

[9] Skolnick, E. 2014. Video Game Storytelling: What Every Developer Needs to Know about Narrative Techniques. New York: Watson-Guptill Publications.

[10]Bryant, R. \& Giglio, K. 2015. Slay the dragon: writing great video games. California: Michael Wiese Productions p. 59

[11] Qin, H. \& Rau, P. \& Salvendy, G. 2009. Measuring Player Immersion in the Computer Game Narrative. Journal of Human-Computer Interaction 25(2), 107-133

[12] Teng, C. 2010. Customization, immersion satisfaction, and online gamer loyalty. Computers in Human Behavior 26, 1547-1554.

[13] Ibister, K. 2006. Better Game Characters by Design : A Psychological Approach. Ebook. Florida: CRC Press. Available at: https://ebookcentral.proquest.com

[14]Calleja, G. 2014. In-Game : From Immersion to Incorporation. Ebook. London: The MIT Press. Available at: https://ebookcentral.proquest.com p.150

[15] Sylvester, T. 2013. Designing Games A Guide to Engineering Experiences. California: O’Reilly p. 84

[16] Collins, K. 2013. Playing with Sound: A Theory of Interacting with Sound and Music in Video Games. Ebook. London: The MIT Press. Available at: https://ebookcentral.proquest.com p58 